\title{
PERFORMANCE ANALYSIS OF NEW PROPOSED WINDOW FOR THE IMPROVEMENT OF SNR \& FIGURE OF MERIT
}

\author{
Mousumi Karmakar ${ }^{1}$, Priyanka Das ${ }^{2}$ \\ ${ }^{1}$ Assistant Professor of Electronics and Communication Engineering \\ Mallabhum Institute of Technology, P.S: Bishnupur, Dist: Bankura-722122, W.B.,India \\ mkmit2008@gmail.com \\ ${ }^{2}$ Assistant ProfessorofElectronics and Communication Engineering \\ Mallabhum Institute of Technology, P.S: Bishnupur, Dist: Bankura-722122, W.B.,India \\ daspriya13@gmail.com
}

\begin{abstract}
The process of communication becomes quite challenging because of the unwanted electrical signals in a communications system. These undesirable signals, usually termed as noise, are random in nature and interfere with the message signals. As a result the signal which is collected from receiver side is not accurate level .In this respects, filtering of signal is very important because noisy signal can mask some important features of the message signal. Hence it is desirable to reduce this noise for proper analysis of the message signal. The signal to noise ratio (SNR) is one of the important measures for reducing the noise. This paper presents the study of low pass FIR filter using a new window techniques for signal Processing. The newly designed windowing filterpresents a new concept for better signal analysis and disturbance detection in the communication systems.The parameters i.e. Power Spectral Density (PSD), signal to noise ratio (SNR), Error Vector Magnitude (EVM) \& Figure of Merit are calculated of original signal and analysis the performance of new proposed window method used for low pass FIR filter. This new windowing filter finds applications in signal analysis, communication system and image compression with a lot of other fields. The results have been concluded using MATLAB R2012 software.
\end{abstract}

Index Terms: Window method for Noise reduction, Figure of Merit, SNR, EVM, New window performance

\section{INTRODUCTION}

A signal as referred to in communication systems, signal processing, and electrical engineering "is a function that conveys information about the behaviour or attributes of some phenomenon" [1]. In the physical world, any quantity exhibiting variation in time or variation in space is potentially a signal that might provide information on the status of a physical system, or convey a message between observers, among other possibilities [2]. The information in a signal is usually accompanied by noise.

The term noise usually means an undesirable random disturbance, but is often extended to include unwanted signals conflicting with the desired signal. These unwanted signals arise from a variety of sources which may be considered in one of two main categories:-

a) Interference, usually from a human source

b) Naturally occurring random noise.

Interference arises for example, from other communication systems (cross talk), $50 \mathrm{~Hz}$ supplies and harmonics, switched mode power supplies, thyristor circuits, ignition (car spark plugs) motors etc. Naturally occurring external noise sources include atmosphere disturbance (e.g. electric storms, lighting, ionospheric effect etc), so called 'Sky Noise' or Cosmic noise which includes noise from galaxy, solar noise and 'hot spot' due to oxygen and water vapour resonance in the earth's atmosphere. These sources can seriously affect all forms of radio transmission and the design of a radio system (i.e. radio, TV, satellite) must take these into account.In recent years, several methods of filtering techniques are used for noise reduction.

In this paper, we have designed a new window technique of FIR low pass filter for the improvement of SNR. In both digital filter design and spectral estimation, the choice of a windowing function can play an important role in determining the quality of overall results. The main role of the window [4],[12] is to damp out the effects of the Gibbs phenomenon that results from truncation of an infinite series.

\section{NEW WINDOW FUNCTION}

In this section new window function is presented. It is defined as [3],[4] 


$$
\begin{gathered}
\mathrm{w}(\mathrm{n})=0.58-0.66 \cos \frac{2 \pi n}{N-1}+0.088 \cos \frac{4 \pi n}{N-1} \\
\text { for } n=0 \text { to } N-1
\end{gathered}
$$

The new window sequence for $\mathrm{N}=63$ \&it's frequency response are presented in Fig. 1 \& 2 respectively with the help of MATLAB 2012 software.

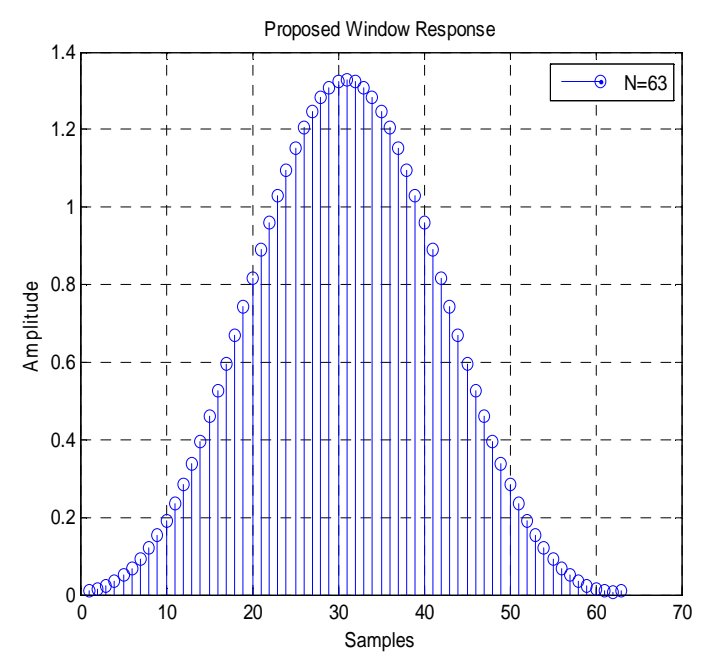

Fig.1: Proposed Window Response

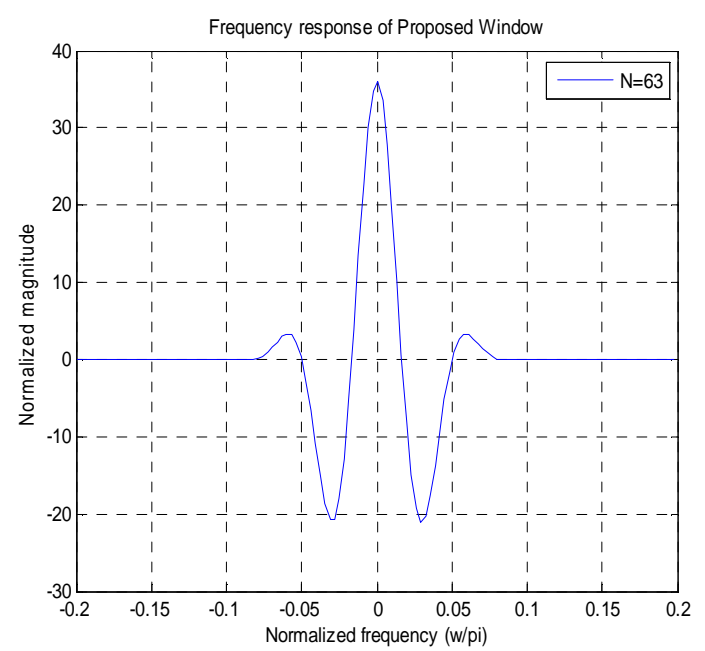

Fig.2: Frequency response of proposed window

\section{CASE STUDY}

It is seen that the noise in communication systems is mostly additive and affects the transmission of signal through a channel. The objective of this paper is to remove this random noise from the communication system $\&$ produce a better result. Now from this point of view a new window method is designed for filtering purpose to get more signal power than noise power at the output.In this section random noise is added to a sinusoidal signal and then passed through a FIR low pass filter using new window function which is shown in Fig.3.

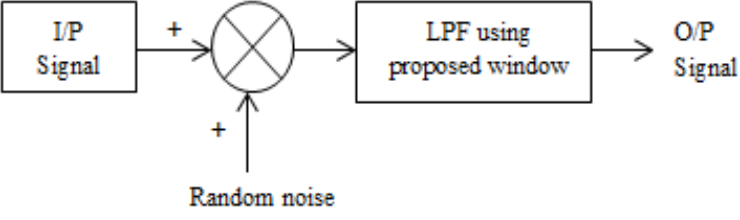

Fig -3: Block diagram of signal transmission

To check the performance of this new window method, two signal i.e. noisy sinusoidal signal \& filtered signal is taken [5]. The simulation is done in MATLAB 2012 software.

\subsection{Simulation Result}

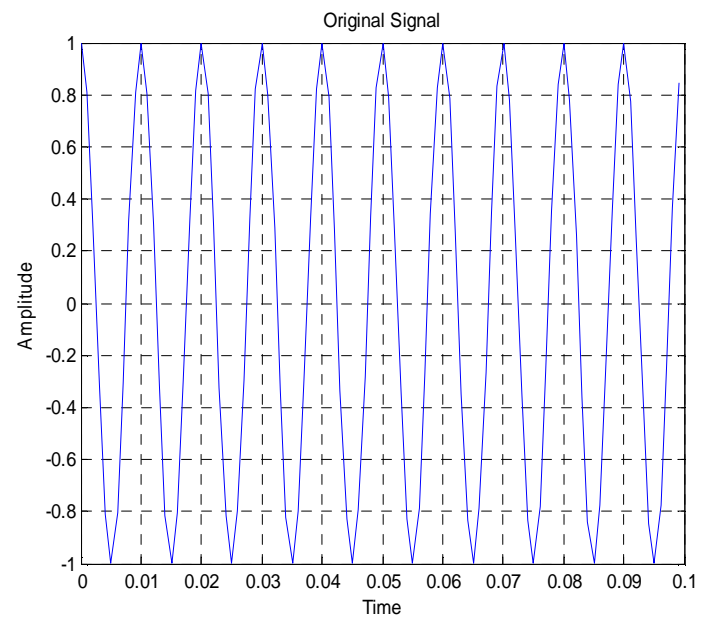

Fig.4: Original Signal

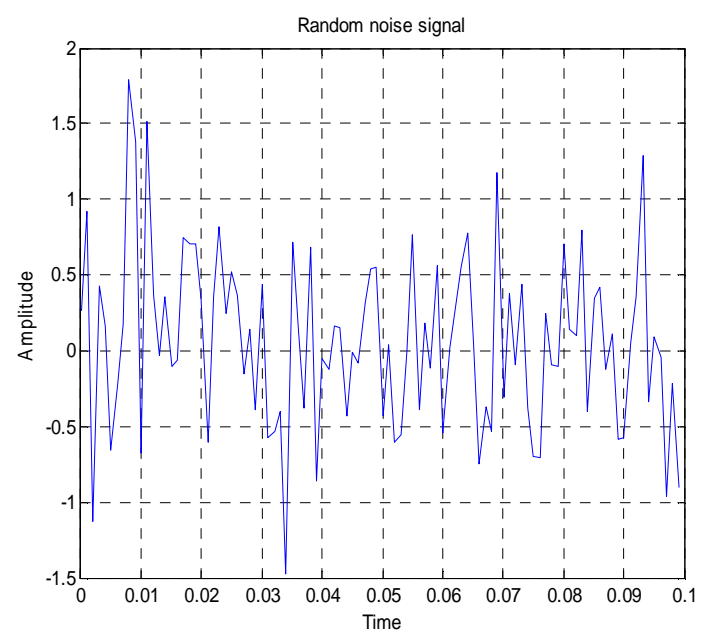

Fig.5: Random noise signal

Input signal i.e. sinusoidal \& random noise signal is shown in Fig. 4 \& 5 respectively. When this two signals is added it becomes noisy \& the characteristics of the sinusoidal is distorted. In general, a high "signal-to-noise ratio" at the output is achieved by passing the noisy signal through a filter. 


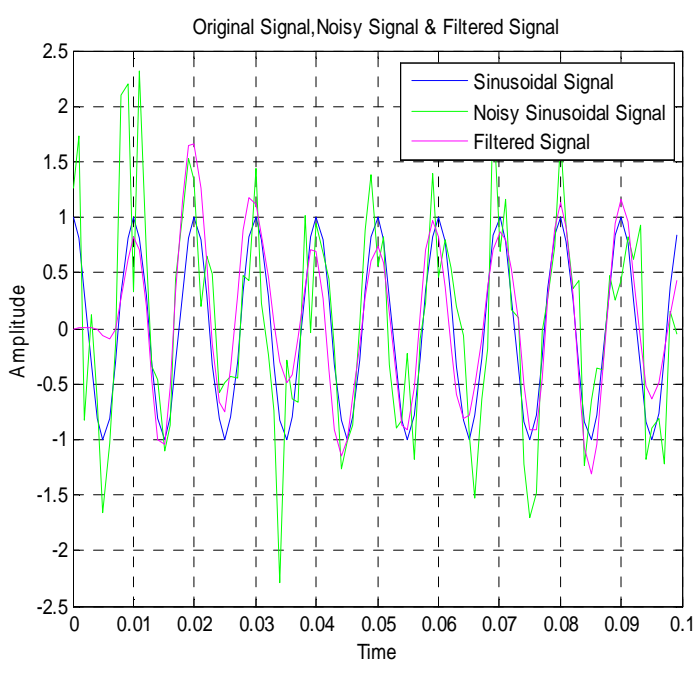

Fig.6: Comparative analysis of original, noisy \& filtered signals

From Fig. 6 it is seen that due to filtering process the noise is almost reduced $\&$ the filtered output is within desired level. The value which is collected from this simulation result is shown in Table. 1

Table.1: Comparative Study of Signals

\begin{tabular}{|c|c|c|}
\hline Parameters & $\begin{array}{c}\text { Noisy Sinusoidal } \\
\text { Signal } \\
\text { (I/P) }\end{array}$ & $\begin{array}{c}\text { Filtered } \\
\text { Signal } \\
\text { (O/P) }\end{array}$ \\
\hline SNR (dB) & 4.658 & 6.942 \\
\hline EVM (\%) & 58.49 & 44.97 \\
\hline
\end{tabular}

From Table. 1 it is clear that the value of filtered output SNR is increased compared to noisy signal SNR whereas error vector magnitude $(\mathrm{EVM})$ is decreased. Mathematically EVM is expressed as [6]:

$\mathrm{EVM}=\frac{1}{\sqrt{S N R}} \times 100 \%$

Generally, if SNR increases by a factor of $\mathrm{N}$, the EVM reduces by $\sqrt{N}$, thus leading to more reliable performance of filter.

\subsection{Figure of Merit}

To get a quantitative measure on how well a given system is "really" doing we have calculated the figure of meritas a ratio of signal-to-noise ratio [7] as follows:

Figure of Merit $=\frac{(S N R)_{o}}{(S N R)_{i}}$

Where, $(\mathrm{SNR})_{\mathrm{o}}[13]$ is the ratio of the average signal power over the average noise power at the output of the filter
$\&(\mathrm{SNR})_{\mathrm{i}}$ is the ratio of the average signal power over the average power of the noise at the input of filter. For a system to be capable of detecting a signal and effective in eliminating noise, it's figure of merit should be high. In this section figure of merit is equal to 1.49 , which is calculated using equation(3).

\subsection{Power Spectral Density(PSD)}

Power spectral density function (PSD) [8] shows the strength of the variations(energy) as a function of frequency. In other words, it shows at which frequencies variations are strong and at which frequencies variations are weak. PSD is a very useful tool to identify oscillatory signals from time series data and to know their amplitude. The unwanted vibrations can be detected from PSD of a signal.

The PSD of noisy signal \& filtered signal using MATLAB simulation[9] is shown in Fig.7.

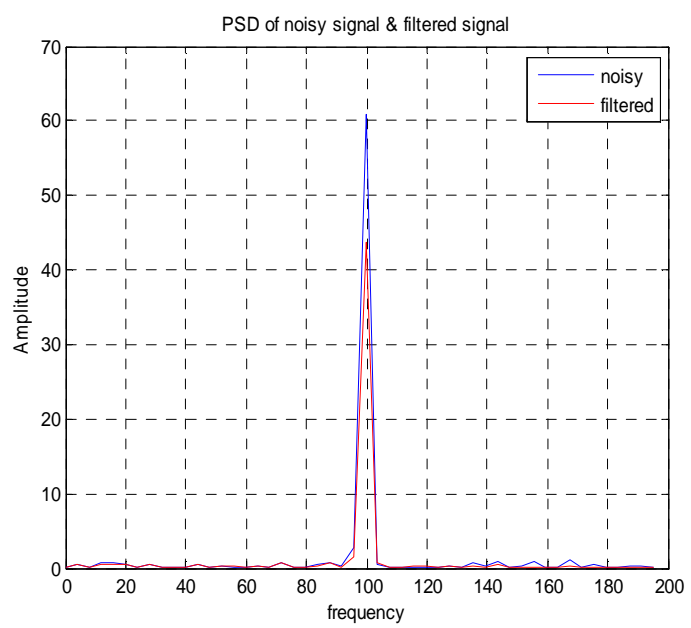

Fig.7: PSD of noisy signal \& filtered signal

From Fig.7 it is seen that only at $100 \mathrm{~Hz}$ the peak amplitude is high, both for noisy \& filtered signal. On the other hand there is no peak at the other frequencies. So this is the peak of original signal. Here it is also clear that, at higher frequency (more than $100 \mathrm{~Hz}$ ) ripples are negligible for filtered signal compared to noisy signal.

\section{APPLICATION OF NEW WINDOW IN COMMUNICATION SYSTEM}

Filters are widely employed in signal processing and communication systems in applications such as channel equalization, noise reduction, radar, audio processing, video processing, biomedical signal processing, and analysis of economic and financial data [10]. The primary functions of filters are one of the followings:

(a) To confine a signal into a prescribed frequency band as in low-pass, high-pass, and band-pass filters.

(b) To modify the frequency spectrum of a signal as in telephone channel equalization and audio graphic equalizers. 
(c) To model the input-output relationship of a system such as telecommunication channels, human vocal tract, and music synthesizers.

Already we have checked that this new window function is capable to remove noise from the noisy signal at the output shown in Fig.6. In this section we have applied this new window for the improvement of SNR and reduction of noise at the output of demodulator of communication system which is shown in Fig.8.

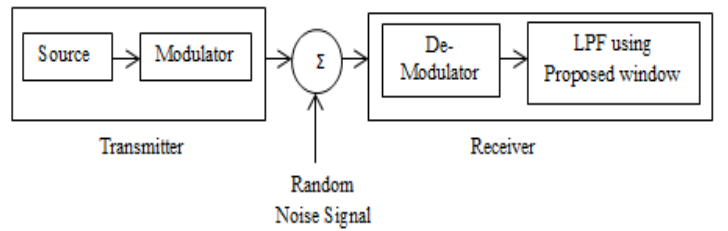

Fig.8: Block diagram of communication system

The simulation is done in MATLAB 2012 software.

\subsection{Simulation Result}

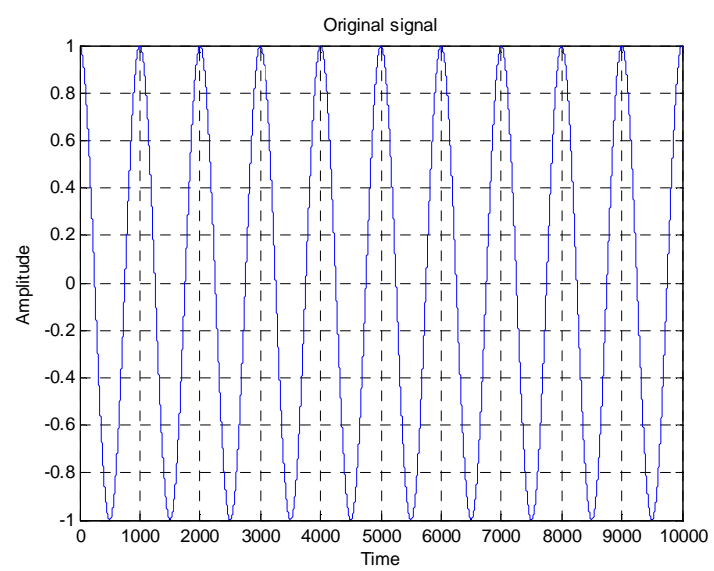

Fig.9: Original message signal

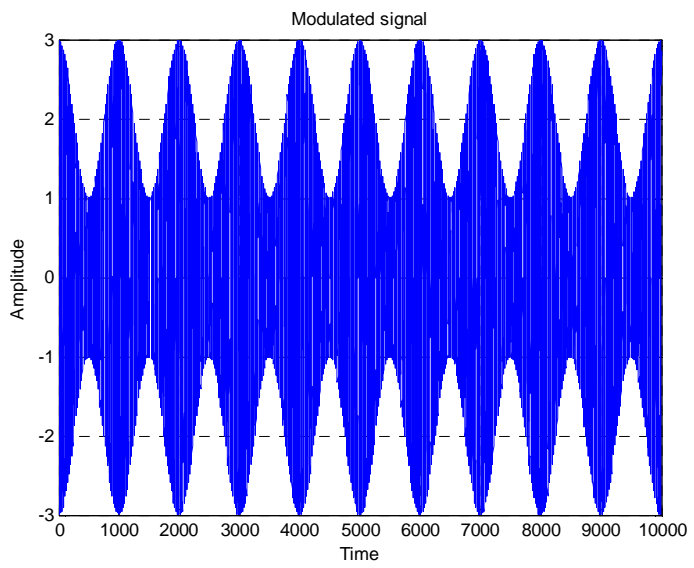

Fig.10: Modulated signal

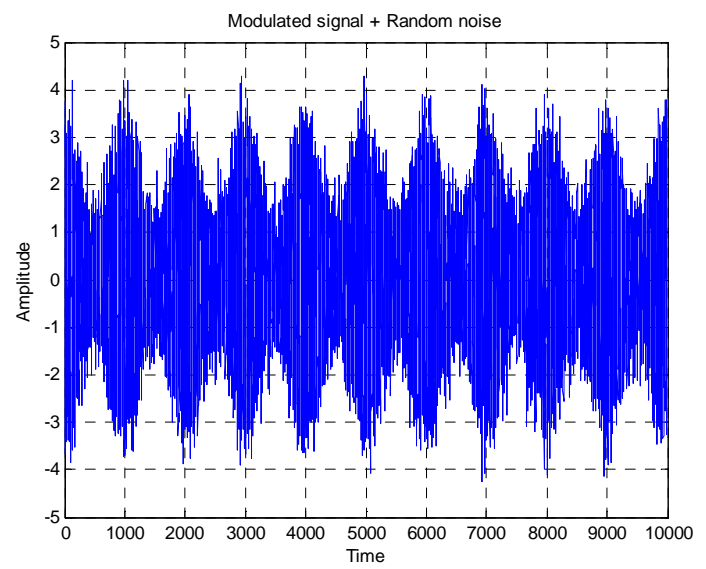

Fig.11: Noisy modulated signal

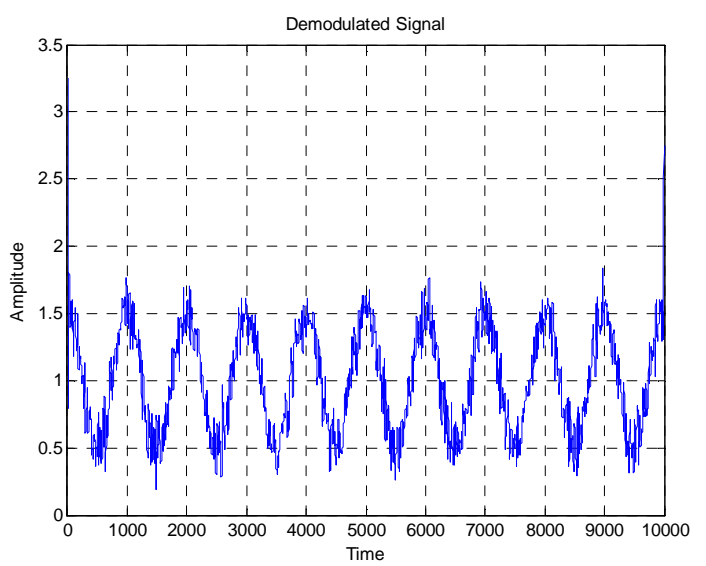

Fig.12: Noisy demodulated signal

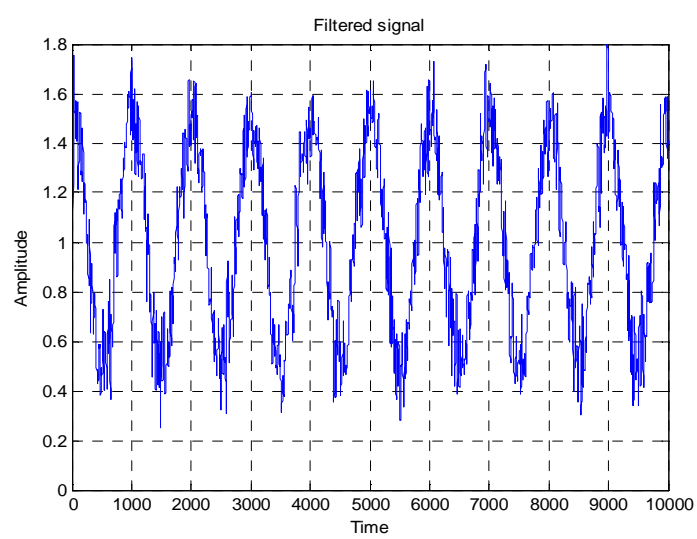

Fig.13: Filtered signal

The value which is collected from this simulation result is shown in Table. 2 
Table.2: Comparative study of noisy signal \& filtered signal

\begin{tabular}{|c|c|c|}
\hline Parameters & $\begin{array}{c}\text { Noisy } \\
\text { Modulated } \\
\text { Signal } \\
\text { (I/P) }\end{array}$ & $\begin{array}{c}\text { Filtered } \\
\text { Demodulated } \\
\text { Signal } \\
\text { (O/P) }\end{array}$ \\
\hline SNR (dB) & 10.109 & 17.716 \\
\hline EVM (\%) & 31.23 & 13.01 \\
\hline
\end{tabular}

Here also to check the performance of the new window function we have calculated the figure of merit (FOM) using equation(3).

Figure of Merit $=\frac{(S N R)_{o}}{(S N R)_{i}}=1.75$

FOM as defined above provides a normalized (SNR)o performance of the modulation-demodulation schemes and larger the value of FOM, better is the performance of the communication system[11] for noise reduction.

\subsection{Performance Analysis}

Comparing Fig.9,12 and 13 it is seen that filtered signal is more similar to original signal. The filtered signal has almost same nature like message (original) signal where as in case of demodulated signal, the features of original signal is distorted somewhere due to random noise. This distortion is removed through FIR low pass filter using new window function (equ.1).

Thus it is proved that this new window method is suitable for FIR filter design. Instead of commonly used window like rectangular, hamming, blackman etc. the new window can be used for any FIR filter design.

\section{CONCLUSION}

Now-a-days, noises are common in communication channels and the recovery of the transmitted signals from the communication path without any noise is considered as one of the difficult tasks. Various denoising technique have been proposed till date for the removal of noises from the transmitted signals. Yet, the effectiveness of those techniques remains an issue. In this paper a new window filter is presented at receiver side for the improvement of SNR and reduction of noise in communication system.

There are several parameters that evaluate the noise performance of any communication system. The first parameter that gives the idea about the efficiency of the system in detecting a signal from a background noise is SNR. The higher value of output SNR than input SNR is significantly verified the better performance of new window filter. The second parameter that evaluates the reliability of receiver is error vector magnitude (EVM). The new approach reduces the EVM at receiver output means more reliable system performance in term of noise reduction. The third parameter that gives the idea about the capability of noise elimination at receiver of the communication system is figure of merit (FOM). For an ideal receiver system figure of merit should be greater than or equal to unity. Here it is seen that figure of merit for filtered signal is greater than unity. Thus the improvement of performance for the new window filter at receiver was experimentally verified.

\section{REFERENCES}

[1] Roland Priemer (1991). Introductory Signal Processing.World Scientific.p. 1.ISBN 9971509199.

[2] Speech processing in embedded systems. Springer.p. 9.ISBN 0387755802.

[3] "A New Window Function to Design FIR Filter with an Improved Frequency Response for Suppressing Side-Lobe Attenuation and Study Comparison with the Other Windows" by Priyanka Das and MousumiKarmakar, International Journal of Engineering Research \& Technology (IJERT),Vol. 2 Issue 12, December - 2013, ISSN: 2278-0181

[4] Oppenheim, A.V., and R.W. Schafer. Discrete-Time Signal Processing. Upper Saddle River, NJ: Prentice-Hall, 1999, p. 468.Oppenheim, A.V., and R.W. Schafer, DiscreteTime Signal Processing, Prentice-Hall, 1989, pp. 447-448.

[5] 'Communication Systems Analog \& Digital' second edition by R P Singh, S D Sapre; The McGraw-Hill Companoes.

[6] R.A. Shafik et al., "On the Error Vector Magnitude as a Performance Metric and Comparative Analysis," 2nd Int. Conf.Emerging Technol., Nov. 2006, pp. 27- 31.

[7] Fundamentals of RF and Microwave Noise Figure Measurements - App.Note 57-1 - Agilent

[8] John G. Proakis, Digital Communications, Mc Graw Hill, third edition, 1995.

[9] FFT for Spectral Analysis Demo - MathWorks India.htm

[10]L.R.Rabiner and B.Gold, Theory and Application of Digital Signal Processing. Englewood Cliffs, NJ: PrenticeHall 1975.

[11] Noise Performance of Various Modulation Schemes by Prof. V. Venkata Rao

[12] John G. Proakis and Dimitris G. Manolakis, Digital Signal Processing, Prentice-Hall, third edition, 1996.

[13] 'Improvement of Noise Performance in Phased-Array Receivers' by Junghyun Kim, Jinho Jeong, and Sanggeun Jeon;

ETRI Journal, Volume 33, Number 2, April 2011 


\section{BIOGRAPHIES}

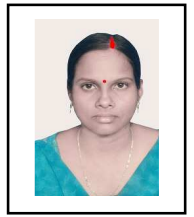

MousumiKarmakar received B.E (2005) degree in Electronics and Communication Engineering from University Institute of Technology, Burdwan University. She obtained M.Tech (2008) in Mechatronics Engg. from NITTTR, Salt-lake, kolkata,West Bengal University of Technology. She is presently working as an Asst. Professor of Department of E.C.E atMallabhum Institute of Technology, Bishnupur, Bankura-722122, W.B., India.Her area of interests includesSignals \& Systems, DSP,Microprocessors \&Microcontrollers,Electronics Circuit design etc. She has an International Journal publication on FIR filter design using window method in IJERT.

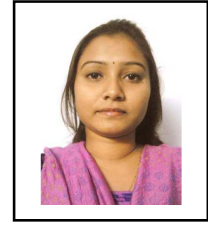

Priyanka Das received B.Tech (2009) degree in Electronics and Instrumentation Engineering \&M.Tech (2011) degree in Mobile Communication \& Networking from JIS College of Engineering, Kalyani, West Bengal University of Technology. She is presently working as an Asst. Professor of Department of E.C.E atMallabhum Institute of Technology, Bishnupur, Bankura-722122, W.B., India. Her area of interests include Signals \& Systems, Digital signal processing, Control System, Electronics Circuit design etc. She has an International Journal publication on FIR filter design using window method in IJERT. 